\title{
Video-Coupled Laser Capture Microdissection Using the PALM MicroBeam: A Powerful Approach for Live Digital Data Communications in Biomedical Research and Education
}

\section{Zheng PP*, van der Weiden M and Kros JM}

Department of Pathology, Erasmus Medical Center, Rotterdam, The Netherlands

Digital data communication has become an important part of various fields in medicine and biology. Laser capture microdissection (LCM) is a useful method for collecting the selected cells for DNA, RNA and protein analysis. LCM can be performed on different types of samples including cryosections, formalin fixed paraffin embedded (FFPE) materials, native tissue, live cells, blood smears, cytologic preparations and chromosomes and more. The advanced LCM with PALM MicroBeam is a state-of-the-art computer-aided light microscopy system enabling contamination-free sampling for isolating cell populations from heterogeneous tissues quickly and reproducibly (Carl Zeiss). It is important to note that the system requires users with essential professional skills for morphological recognition of the target cell populations to achieve the maximum possible precision of the isolated cells. The system is particularly useful for diagnostic pathologists or other professionals. In this study we tested the videocoupled LCM with PALM MicroBeam as a powerful approach for live digital data communication, including real time monitoring, retrieving and analyzing the precision and accuracy of the laser ablated cells in biomedical researches.

The study was conducted with the approval of the institutional ethical review board. Both cryosections $(n=6)$ and paraffin sections $(\mathrm{n}=6)$ from clinical glioma specimens were used. Normal glass slides were oven baked at $150^{\circ} \mathrm{C}$ for at least 6 hours prior to mounting the tissue slices $(5 \mu \mathrm{m})$ on a baked glass slide. The collection tubes are certified free of human DNA, RNase, DNase, and endotoxins (AB-0350, Thermo Fisher Scientific Inc). Generally, the samples used for LCM could be either hematoxylin-eosin (HE) stained, or immunostained, or unstained. HE staining is normally sufficient for recognition of those cell populations with unique morphology by diagnostic pathologists

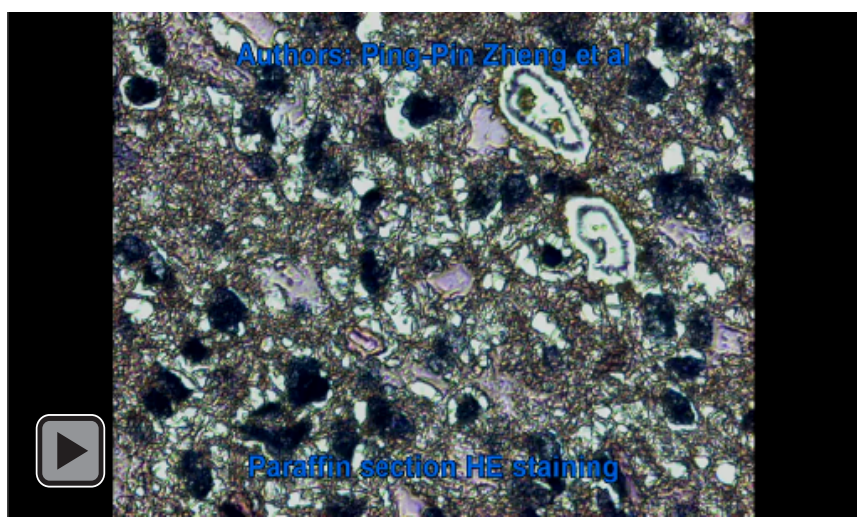

Video 1: Simultaneous isolation of the nuclei and the cytoplasm of individual gemistocytes on paraffin section. Gemistocytes in a human glioma are stained by $\mathrm{HE}$ and visualized under a $63 \mathrm{X}$ objective. Whole cell bodies of the selected individual cells containing the nucleus and cytoplasm were captured and micro-ablated by PALM MicroBeam. The video data can be accessed by using QuickTime.

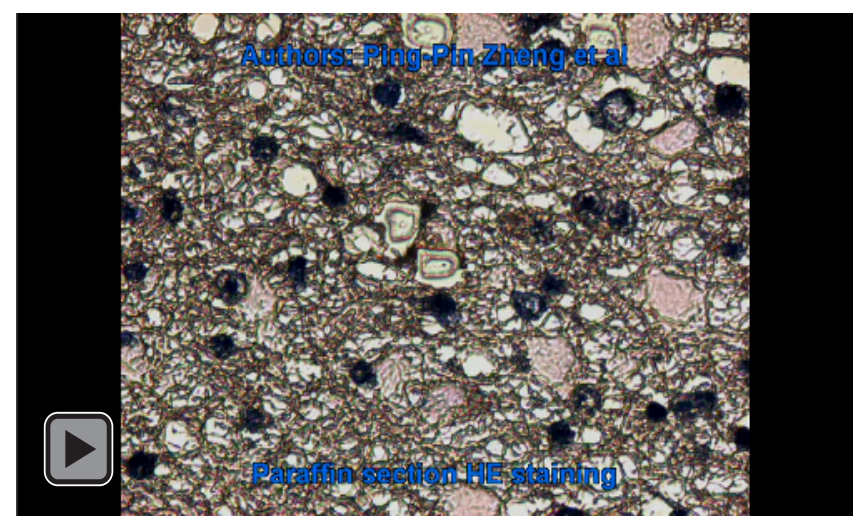

Video 2: Isolation of the nuclei of individual gemistocytes on paraffin section. Gemistocytes in a human glioma are stained by HE and visualized under a $63 \mathrm{X}$ objective. The nuclei of the selected gemistocytes are selectively captured and micro-ablated by PALM MicroBeam. The video data can be accessed by using QuickTime.

or other morphologists, e.g. gemistocytes, multinucleated giant cells, epithelial cells, myocytes, neurons, osteocytes and so on. In contrast to $\mathrm{HE}$ or unstained samples, immunostaining facilitates the selection precision for isolating the targeted cell population. While it is important to realize that the approach is costly and time-consuming. It is possible to increase the risk of degradation of nucleic acids and/ or proteins during the staining processes. Precaution should be taken in this regards prior to use. However, immunostaining is required for isolating cells with ill-defined morphology which are not precisely detected by routine HE stain.

Purification of selected cell populations from heterogeneous samples prior to further investigations is important for cell-lineage specific analysis in biomedical research. Particularly in cancer research targeting of cells of interest is important because contamination from unwanted cells may shift the results and conceal the signals of the relevant cells. DNA/RNA/protein isolation and sequencing from unselected/mixed cell populations pollute molecular profiles

*Corresponding author: Ping-Pin Zheng, Department of Pathology, Erasmus Medical Center, Office JNI Be262a, Dr. Molewaterplein 50, PO Box 1738, 3000 DR, Rotterdam, The Netherlands, E-mail: p.zheng.1@erasmusmc.nl

Received August 06, 2013; Accepted August 08, 2013; Published August 13 2013

Citation: Zheng PP, van der Weiden M, Kros JM (2013) Video-Coupled Laser Capture Microdissection Using the PALM MicroBeam: A Powerful Approach for Live Digital Data Communications in Biomedical Research and Education. J Mol Genet Med 7: 65. doi:10.4172/1747-0862.1000069

Copyright: $\odot 2013$ Zheng PP, et al. This is an open-access article distributed under the terms of the Creative Commons Attribution License, which permits unrestricted use, distribution, and reproduction in any medium, provided the original author and source are credited 
Citation: Zheng PP, van der Weiden M, Kros JM (2013) Video-Coupled Laser Capture Microdissection Using the PALM MicroBeam: A Powerful Approach for Live Digital Data Communications in Biomedical Research and Education. J Mol Genet Med 7: 65. doi:10.4172/17470862.1000069

Page 2 of 3

among different cell populations [1] rather than sharply unraveling the molecular characteristics from a unique cell population. PALM MicroBeam system (Carl Zeiss) empowers researchers to precisely target and select cell populations, and allows micromanipulation down to the subcellular level. In this study, we show the live processes of isolation of microglia and gemistocytes from human glioma samples by video-coupled LCM with PALM MicroBeam. To achieve a high-resolution visualization of the selected cell populations, the microscope objective was set at $63 \mathrm{x}$ magnification for visualization of all the tissue sections used in this study. Dependent on the nature of subsequent experiments, the selected cells could be isolated entirely containing cytoplasm and nuclei (Videos 1,4), or alternatively nuclei (Video 2) or cytoplasm (Video 3) are focused. Gemistocytes have unique morphological features consisting of abundant cytoplasm and an eccentric nucleus, and may contain two or more nuclei in the cell, and they are readily to be recognized in HE stained sections (Videos 1-3). Gemistocytes are absent in normal central nervous system (CNS), but appear in a variety of CNS diseases including gliomas, leukoencephalopathy, reactive gliosis in infection, inflammation and demyelinating disease [2]. Gemistocytes are generally considered as reactive and/or neoplastic astrocytes characterized by expression

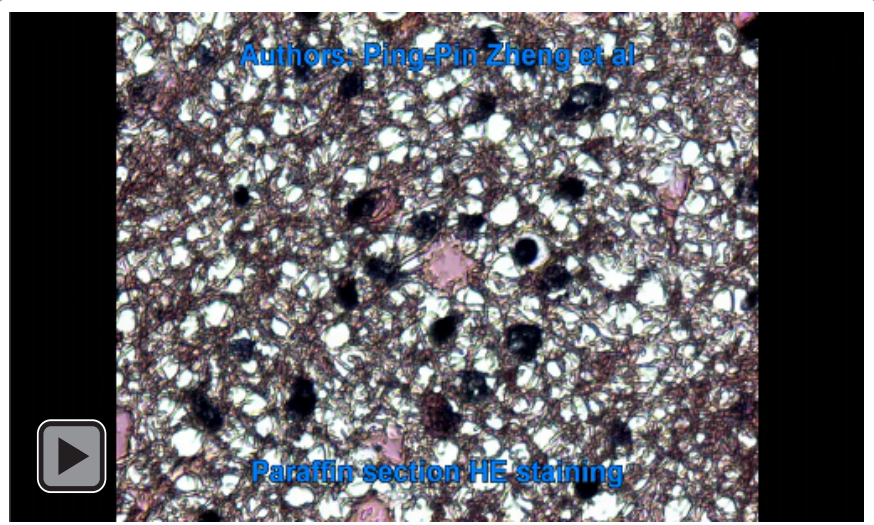

Video 3: Isolation of the cytoplasm of individual gemistocytes on paraffin section. Gemistocytes in a human glioma are stained by HE and visualized under a $63 \mathrm{X}$ objective. The Cytoplasm of the selected gemistocytes is selectively captured and micro-ablated by PALM MicroBeam. The video data can be accessed by using QuickTime.

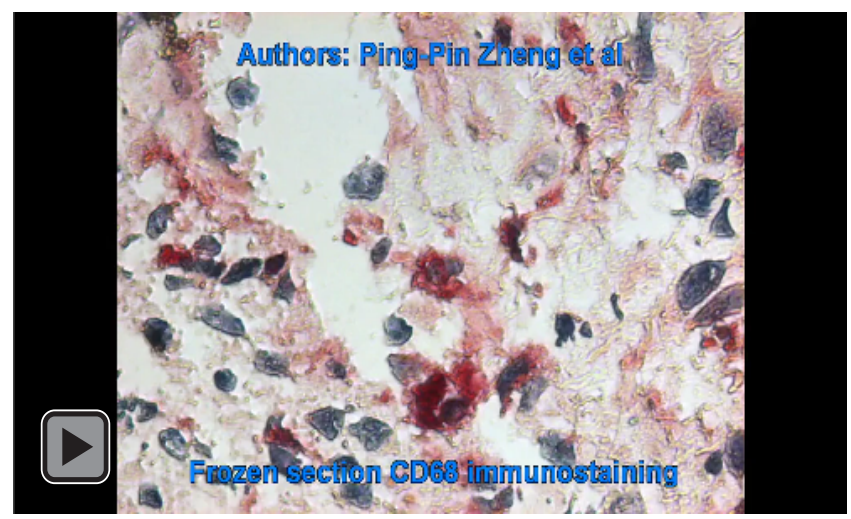

Video 4: Isolation of CD68 labelled microglia/macrophages on frozen section. In a frozen section from a human glioma, microglia/macrophages are immunolabelled with CD68 and visualized under a $63 \mathrm{X}$ objective. The selected cells containing the nuclei and the cytoplasm are captured and micro-ablated by PALM MicroBeam. The video data can be accessed by using QuickTime.

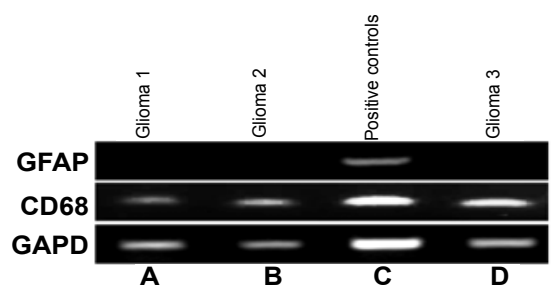

Figure 1: CD68-immunopositive cells isolated by LCM with PALM express CD68 mRNA but not GFAP as assessed by RT-PCR. GAPD is used as internal control. The positive controls for expression of CD68 (human peripheral blood leukocytes) and GFAP (human autopsy brain) are included in the RT-PCR experiments as shown in panel $\mathrm{C}$. The obtained CD68-immunopositive cells by LCM with PALM express CD68 mRNA but not GFAP as shown in panels A, B and $\mathrm{D}$.

of glial fibrillary acidic protein (GFAP) [3]. While they also express major histocompatibility complex (MHC) class II molecules [4], a well-known family of molecules normally found only on immune cells. Further investigations using homogeneous populations of gemistocytes are required for addressing the origin(s) and biological role(s) of gemistocytes in various categories of CNS diseases and most importantly in brain tumours. Microglia are the resident macrophages in CNS and are implicated in almost all neuropathological processes, including CNS inflammation, neurodegenerative diseases and brain tumours. Morphologically microglia demonstrate a versatile spectrum of the phenotypes [5]. HE staining is usually insufficient for detection of the full spectrum of microglial phenotypes. We used CD68 as before [6] for identifying microglia/macrophages (Video 4). A validation experiment by RT-PCR was used for confirmation of the purity from the LCM-derived cells. For example: the source materials obtained from the CD68-immunopositive cells express CD68 mRNA but not GFAP (Figure 1). Extraction of RNA and generation of cDNA were as described before $[7,8]$. The sequences of the primer sets as described elsewhere for CD68 [9] and for GFAP [10]. Glyceraldehyde 3-phosphate dehydrogenase (GAPD) served as an internal control as in our previous studies $[7,8]$. All of the primers used in this study were commercially synthesized (Invitrogen, Carlsbad, CA).

In conclusion, the study shows that video-coupled LCM with PALM MicroBeam is very powerful approach for live digital data communication, including monitoring, retrieving and analyzing real time data in biomedical research, but also it could be useful as educational tools for digitally training of non-professional users for efficiently using the system.

\section{Competing Interests}

The authors declare that they have no competing interests.

\section{References}

1. Heng HH, Bremer SW, Stevens JB, Ye KJ, Liu G, et al. (2009) Genetic and epigenetic heterogeneity in cancer: a genome-centric perspective. J Cell Physiol 220: 538-547.

2. Tihan T, Vohra P, Berger MS, Keles GE (2006) Definition and diagnostic implications of gemistocytic astrocytomas: a pathological perspective. J Neurooncol 76: 175-183.

3. Kros JM, Waarsenburg N, Hayes DP, Hop WC, van Dekken H (2000) Cytogenetic analysis of gemistocytic cells in gliomas. J Neuropathol Exp Neurol 59: 679-686.

4. Geranmayeh F, Scheithauer BW, Spitzer C, Meyer FB, Svensson-Engwall AC et al. (2007) Microglia in gemistocytic astrocytomas. Neurosurgery 60: 159166 
Citation: Zheng PP, van der Weiden M, Kros JM (2013) Video-Coupled Laser Capture Microdissection Using the PALM MicroBeam: A Powerful Approach for Live Digital Data Communications in Biomedical Research and Education. J Mol Genet Med 7: 65. doi:10.4172/17470862.1000069

Page 3 of 3

5. Tambuyzer BR, Ponsaerts P, Nouwen EJ (2009) Microglia: gatekeepers of central nervous system immunology. J Leukoc Biol 85: 352-370.

6. Zheng PP, van der Weiden M, van der Spek PJ, Vincent AJ, Kros JM (2012) Isocitrate dehydrogenase $1 \mathrm{R} 132 \mathrm{H}$ mutation in microglia/macrophages in gliomas: indication of a significant role of microglia/macrophages in glial tumorigenesis. Cancer Biol Ther 13: 836-839.

7. Zheng PP, van der Weiden M, van der Spek PJ, Vincent AJ, Kros JM (2013) Intratumoral, not circulating, endothelial progenitor cells share genetic aberrations with glial tumor cells. J Cell Physiol 228: 1383-1390.

8. Zheng PP, Sieuwerts AM, Luider TM, van der Weiden M, Sillevis-Smitt
PA, et al. (2004) Differential expression of splicing variants of the human caldesmon gene (CALD1) in glioma neovascularization versus normal brain microvasculature. Am J Pathol 164: 2217-2228.

9. Ding $Q$, Jin $T$, Wang $Z$, Chen $Y$ (2007) Catalase potentiates retinoic acidinduced THP-1 monocyte differentiation into macrophage through inhibition of peroxisome proliferator-activated receptor gamma. J Leukoc Biol 81: 15681576.

10. Schwartz PH, Bryant PJ, Fuja TJ, Su H, O'Dowd DK, et al. (2003) Isolation and characterization of neural progenitor cells from post-mortem human cortex. J Neurosci Res 74: 838-851 